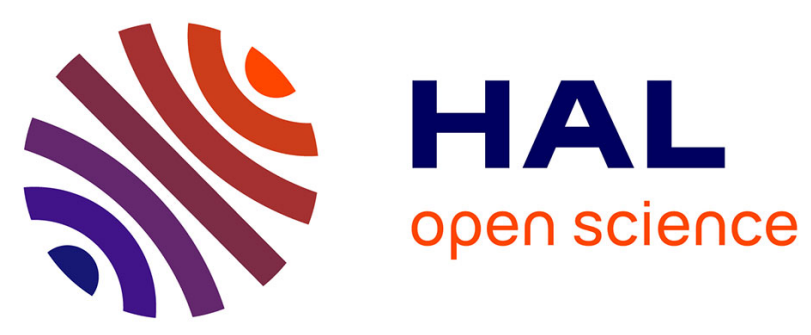

\title{
The impact of social isolation and changes in work patterns on ongoing thought during the first COVID-19 lockdown in the United Kingdom
}

Brontë Mckeown, Giulia Poerio, Will Strawson, Léa Martinon, Leigh Riby, Elizabeth Jefferies, Cade Mccall, Jonathan Smallwood

\section{To cite this version:}

Brontë Mckeown, Giulia Poerio, Will Strawson, Léa Martinon, Leigh Riby, et al.. The impact of social isolation and changes in work patterns on ongoing thought during the first COVID-19 lockdown in the United Kingdom. Proceedings of the National Academy of Sciences of the United States of America, 2021, 118 (40), pp.e2102565118. 10.1073/pnas.2102565118 . hal-03366024

\section{HAL Id: hal-03366024 https://hal.science/hal-03366024}

Submitted on 25 Oct 2021

HAL is a multi-disciplinary open access archive for the deposit and dissemination of scientific research documents, whether they are published or not. The documents may come from teaching and research institutions in France or abroad, or from public or private research centers.
L'archive ouverte pluridisciplinaire HAL, est destinée au dépôt et à la diffusion de documents scientifiques de niveau recherche, publiés ou non, émanant des établissements d'enseignement et de recherche français ou étrangers, des laboratoires publics ou privés. 


\section{PNAS \\ www.pnas.org}

2 Main Manuscript for

3 The impact of social isolation and changes in work patterns on ongoing thought during the first

4 COVID-19 lockdown in the UK.

5 Brontë Mckeown ${ }^{a}$, Giulia L. Poerio ${ }^{b}$, Will H. Strawson ${ }^{c}$, Léa M. Martinon ${ }^{d}$, Leigh M. Riby ${ }^{e}$, 6 Elizabeth Jefferies ${ }^{a}$, Cade McCall ${ }^{a 1}$, Jonathan Smallwood ${ }^{\text {f1 }}$

$7 \quad{ }^{a}$ Department of Psychology, University of York, YO10 5DD, United Kingdom

${ }^{\mathrm{b}}$ Department of Psychology, University of Essex, CO4 3SQ, United Kingdom

${ }^{\mathrm{c}}$ Neuroscience, Brighton and Sussex Medical School, University of Sussex, BN1 9PX, United 10 Kingdom

${ }^{d}$ Laboratoire de Psychologie Cognitive et Sociale (LAPSCO) CNRS UMR 6024, Université

12 Clermont Auvergne, 63000 Clermont-Ferrand, France

$13{ }^{e}$ Department of Psychology, Northumbria University, NE1 8ST, United Kingdom

14 f Psychology Department, Queen's University, K7L 3N6, Canada

15 Corresponding author: Brontë Mckeown, bronte.mckeown@york.ac.uk

16 Author Contributions: Brontë Mckeown: designed research, prepared materials, performed research, analyzed data, visualization, writing- original draft, writing- review and editing. Giulia L. Poerio: designed research, prepared materials, performed research, writing- review and editing. Will H. Strawson: designed research, prepared materials, writing- review and editing. Léa M. Martinon: performed research, writing- review and editing. Leigh M. Riby: designed research, funding acquisition, writing- review and editing. Elizabeth Jefferies: designed research, supervision, funding acquisition, writing- review and editing. Cade McCall: designed research, prepared materials, writing- original draft, writing- review and editing, supervision. Jonathan Smallwood: designed research, writing- original draft, writing-review and editing, supervision, funding acquisition.

26 Competing Interest Statement: None.

27 Classification: Social sciences, Psychological and Cognitive Sciences

28 Keywords: COVID-19, Lockdown, Isolation, Thoughts, Experience-sampling

${ }^{1}$ Joint senior author 
This PDF file includes:

\author{
Main Text \\ Figure legends 1 to 3 . \\ Figures 1 to 3 .
}

\begin{abstract}
\end{abstract}
The COVID-19 pandemic led to lockdowns in countries across the world, changing the lives of billions of people. The UK's first national lockdown, for example, restricted people's ability to socialize and work. The current study examined how changes to socializing and working during this lockdown impacted ongoing thought patterns in daily life. We compared the prevalence of thought patterns between two independent real-world experience-sampling cohorts, collected before- and during-lockdown. In both samples, young (18-35) and older (55+) participants completed experience-sampling measures five times daily for seven days. Dimension reduction was applied to these data to identify common "patterns of thought". Linear mixed modelling compared the prevalence of each thought pattern (i) before- and during-lockdown, (ii) in different age groups and (iii) across different social and activity contexts. During lockdown, when people were alone, social thinking was reduced, but on the rare occasions when social interactions were possible, we observed a greater increase in social thinking than pre-lockdown. Furthermore, lockdown was associated with a reduction in future-directed problem-solving, but this thought pattern was reinstated when individuals engaged in work. Therefore, our study suggests that the lockdown led to significant changes in ongoing thought patterns in daily life and these changes were associated with changes to our daily routine that occurred during lockdown.

\title{
Significance Statement
}

Since the emergence of COVID-19, lockdowns have been imposed across the globe. These lockdowns change daily life considerably, reducing opportunities to socialize and work. The current study investigated how these changes may impact people's ongoing thought patterns by examining experience-sampling data gathered before and during the UK's first national lockdown. We found that socializing and working were significant predictors of ongoing thought in daily life and that limiting these activities during lockdown contributed to changes in ongoing thought patterns. Our findings highlight how ongoing thought patterns are shaped by the daily activities we engage in, both during lockdowns and in more normal times.

\section{Main Text}

\section{Introduction}

On March 23rd, 2020, the UK entered a nationwide lockdown to curb the spread of COVID-19. This first national lockdown required people to stay at home and not meet with anyone outside their household. Social gatherings were banned, and "non-essential" industries were closed, reducing opportunities for work (1). There were also large economic changes (2), and death rates increased substantially (3). Studies show the lockdown had widespread psychological and behavioral consequences, including elevated anxiety and depression levels (4), overall deterioration of mental health (5), changes to diet and physical activity (6-8), high levels of loneliness (9) and increasing suicidal ideation (10). Our study used experience-sampling to measure patterns of ongoing thoughts before and during lockdown in the UK, with the aim of understanding how specific features of the stay-at-home order impacted people's thinking in daily life, and to use this data to inform contemporary theoretical views on ongoing thought.

Our investigation served three broad goals. First, the lockdown led to changes in opportunities for socializing, and contemporary theories of ongoing thought suggest that social processing is an important influence on our day to day thinking $(11,12)$. For example, previous research indicates that individuals spend a lot of time thinking about other people in daily life $(13,14)$ or when performing tasks dependent on social cognition in the laboratory (15). Importantly, spontaneous social thoughts decline following periods of solitude and increase following periods of social 
interaction in the laboratory (11). They can also facilitate socio-emotional adjustment during important life transitions, such as starting University (16). Furthermore, ongoing thought patterns with social features are associated with increased neural responses to social stimuli (in this case, faces) (17). Such evidence suggests the social environment can shape ongoing thought, leading to the possibility that changes in opportunities for socialization following the stay-at-home order could have changed the expression of social thinking in daily life.

Second, lockdowns also disrupted individuals' normal working practices, forcing people to reassess their goals. Prior work highlights that ongoing thought content is linked to an individual's current concerns and self-related goals (18-21), and experimentally manipulating an individual's goals can prime ongoing thought to focus on these issues (21-23). In particular, a substantial proportion of ongoing thoughts are future-directed $(14,18,21,24-26)$, and this "prospective bias" is thought to support the formation and refinement of personal goals for future behavior $(18,21$, $27,28)$. Notably, this type of thought is also important in maintaining mental health through associations with improved subsequent mood (24) and reduced suicidal ideation $(29,30)$. Changes to opportunities for working during the lockdown, therefore, provide a chance to understand whether prospective features of ongoing thought are altered when important external commitments change.

Third, previous work indicates that the contents of thought vary across the lifespan. For example, during periods of low cognitive demand, younger adults report significantly more future-directed thoughts while older adults report significantly more past-related thoughts (31). At rest, older adults report more 'novel' and present-oriented thoughts compared to younger adults (32). In daily life, older adults tend to report fewer off-task thoughts than younger adults, and their thoughts are rated as more 'pleasant', 'interesting' and 'clear' (33). Finally, aging is associated with a decline in daydreaming, particularly a reduction on topics such as the future, fear of failure, or guilt (34). However, the degree to which these age-related changes are explained by lifestyle differences between young and older individuals is unclear. The lockdown may have altered key contextual factors that, under normal circumstances, differ systematically between younger and older adults. For example, increasing age is associated with more interactions with family members and fewer with 'peripheral partners' (e.g., coworkers, acquaintances, strangers) (35), a pattern that may be common in younger people during lockdown. With all this in mind, the lockdown provided an opportunity to examine whether changes to daily life during lockdown differentially impacted ongoing thought patterns in younger and older individuals.

Our study used an experience-sampling methodology in which people are signaled at random times in their daily lives to obtain multiple reports describing features of their ongoing thoughts and the context in which they occur (e.g., social environment, activity and location) (36). To examine the contents of people's thoughts, we used multidimensional experience-sampling (MDES) (37). In this method, participants describe their in-the-moment thoughts by rating their thoughts on several dimensions (e.g., temporal focus or relationship to self and others) (38). Dimension reduction techniques can then be applied to use covariation in the responses to different questions to identify "patterns of thought" $(37,39)$. Previous studies have used MDES to identify common patterns of ongoing thought, varying in both form and content, often with distinct neural correlates $(28,37,39-43)$. For example, a pattern of episodic social cognition is associated with increased activity within regions of the ventromedial prefrontal cortex associated with memory and social cognition (41), while a pattern of external task focus is associated with increased activity in the intra-parietal sulcus (42). In addition, at rest, visual imagery is associated with stronger interactions between the precuneus and lateral fronto-temporal network (44), while detailed task focus is high during working memory tasks (15) and other complex tasks (45) and linked to activity in the default mode network during working memory maintenance (46).

In summary, our study set out to examine whether ongoing thought patterns experienced during lockdown differed from those normally reported in daily life, focusing on the consequences of changes in opportunities for socialization and work. The pre-lockdown sample was an existing 
dataset used to provide a baseline for ongoing thought patterns in daily life before lockdown restrictions. In both samples, young (18-35) and older (55+) participants completed surveys five times daily over seven days. Each sampling point obtained in the moment measured key dimensions of ongoing thought using MDES (37). Participants also provided information regarding the social environment in which the experience occurred. Dimension reduction was applied to both samples' thought data to identify common "patterns of thought". We then used linear mixed modelling to explore the prevalence of each thought pattern (i) before- and duringlockdown, (ii) in different age groups, and (iii) across social contexts. In the lockdown sample, participants provided additional information regarding their current activity (e.g., working or leisure activities) and virtual social environment, which we used to explore how specific features of daily life during lockdown corresponded with patterns of thought.

\section{Results \\ Changes to daily life during lockdown}

In both samples, after first assessing the contents of their thoughts, participants were asked about their social environment immediately before being signalled. We expected that the percentage of responses for which participants reported being alone would be higher in the lockdown sample than the pre-lockdown sample. To test this, we calculated the percentage of each participant's responses in which they said they were (i) alone, (ii) around people but not interacting and (iii) around people and interacting. Sample means for each of the three percentages, for young and older participants, is shown in Figure 1 (panel A). A two-way ANOVA confirmed that during lockdown, the 'alone' percentage was significantly higher compared to prelockdown $\left(F(1)=12.03, p<.001, \eta_{p}^{2}=0.06\right)$ and significantly higher for younger compared to older participants across both samples (pre- and during-lockdown) $\left(F(1)=13.25, p<.001, \eta_{p}^{2}=\right.$ 0.06). Participants in the lockdown sample also reported their location immediately before completing the survey. Overall percentages for each option are shown in Figure 1 (panel B), revealing that $85 \%$ of responses were "Inside at home". These analyses establish that people spent more time alone during lockdown, and most of their time inside at home.

\section{Patterns of thought}

To identify common "patterns of thought" across both samples, we combined the thought data from both samples (see Table S1) and decomposed these in a single Principal Components Analysis (PCA). Based on eigenvalues $>1$, five components- accounting for $53 \%$ of the total variance- were retained for further analysis (see Fig. S1 for scree plot): (i) 'Future-directed problem-solving'- describing patterns of thought with the highest loadings on 'Problem-solving', 'Future-goals', 'Controlled' and 'Rehearsing-future', (ii) 'Pleasant engagement'- with the highest loadings on 'Positive', 'Wanted', 'Current goals' and 'Task', (iii) 'Episodic social cognition'- with the highest loadings on 'Close-others', 'Important', 'Self' and 'Future', (iv) 'Imagery'- with the highest loadings on 'Vivid', 'Images' and 'Detailed' and (v) 'Detailed task focus'- with the highest loadings on 'Words', 'On-task', 'Detailed' and 'Current goals'. Item loadings on these components are presented as word clouds in Figure 1 (panel C) (see Table S2 for exact component loadings). To ensure that the thought patterns identified across samples were present in both samples, we ran a PCA on each sample separately (specifying five components for extraction) and correlated each participant's PCA score from this analysis with their PCA score from the combined analysis, revealing a high correspondence between patterns seen in the two samples (see Fig. S2 for scatterplots).

\section{Comparing thought patterns between (i) pre- and during-lockdown samples, (ii) age groups and (iii) social environments.}

Having identified five patterns of thought, we examined the influence that lockdown, and changes to social interactions during lockdown, had on ongoing thought by comparison with the baseline group. We performed a series of linear mixed models in which each of the five thought patterns was the outcome measure (see Methods). These models included three explanatory variables and their interactions: (i) whether the sample was pre- or during-lockdown, (ii) whether the 
individual was young or older and (iii) the nature of the social environment in which the experience occurred (alone, with others not interacting, with others and interacting). For each model, alpha was set to < .01 (two-tailed) to account for family-wise error emerging from conducting five models (i.e., .05/5). The reported alpha levels in our paper are unadjusted; main effects and interactions are considered significant only at the $p<.01$ level. When probing these significant main effects and interactions using pairwise comparisons, the alpha level was Bonferroni adjusted to account for the number of tests being conducted; here, the adjusted alpha levels are reported in parentheses. Estimates are unstandardized and reflect the difference between each factor level and the intercept (grand mean of all conditions). These results are summarized in Figure 2 (see Tables S3-S5 for ANOVA output, parameter estimates, and the variance explained by random effects).

Model 1: Future-directed problem-solving. There was a significant main effect of Sample (prevs during-lockdown) $(F(1)=16.19, p<.001)$. Future-directed problem-solving was lower in the lockdown sample $(b=-0.15,95 \% \mathrm{Cl}[-0.23,-0.08], t(191)=-4.02, p<.001)$. There was also a significant main effect of Age-group $(F(1)=6.33, p=.013)$, with future-directed problem-solving higher in younger participants $(b=0.10,95 \% \mathrm{Cl}[0.02,0.17], t(188)=2.52, p=.012)$. There was also a significant main effect of Social environment $(F(2)=31.36, p<.001)$, with future-directed problem-solving lower when interacting with other people $(b=-0.17,95 \% \mathrm{Cl}[-0.21,-0.12]$, $t(4850)=-7.52, p<.001)$. Therefore, the lockdown was associated with a reduction in futuredirected problem-solving, regardless of social environment or age-group.

Model 2: Pleasant engagement. Levels of pleasant engagement significantly varied by Agegroup $(F(1)=19.82, p<.001)$ and was lower in younger participants $(b=-0.19,95 \% \mathrm{Cl}[-0.27$, $0.10], t(191)=-4.45, p<.001)$. There was a significant main effect of Social environment $(F(2)=$ $5.43, p=.004)$, with pleasant engagement highest when participants were interacting with others $(b=0.07,95 \% \mathrm{Cl}[0.03,0.11], t(4833)=3.29, p<.001)$ and lowest when around people but not interacting $(b=-0.05,95 \% \mathrm{Cl}[-0.10--0.00], t(4802)=-1.99, p=.046)$. There was a significant interaction between Age-group and Social environment $(F(2)=5.60, p=.004)$. Pairwise comparisons at each level of Social environment, split by Age-group (Bonferroni adjusted for 6 tests) revealed that for younger participants, pleasant engagement was significantly higher when interacting with other people compared to when alone $(b=0.20,95 \% \mathrm{Cl}[0.09,0.31], t(4808)=$ $4.90, p<.001)$ or when around other people but not interacting $(b=0.22,95 \% \mathrm{Cl}[0.09,0.36]$, $t(4786)=4.43, p<.001)$. For older participants however, pleasant engagement did not significantly vary across social environments ( $p$ 's > .05). Regardless of the lockdown, therefore, social situations were characterized by higher levels of pleasant engagement for younger individuals.

Model 3: Episodic social cognition. There was a significant main effect of Social environment $(F(2)=35.20, p<.001)$ with episodic social cognition highest when interacting with others $(b=$ $0.19,95 \% \mathrm{Cl}[0.14,0.23], t(4840)=8.37, p<.001)$ and lowest when around people but not interacting $(b=-0.11,95 \% \mathrm{Cl}[-0.16--0.06], t(4815)=-4.35, p<.001)$. There was a significant main effect of Age-group $(F(1)=6.10, p=.014)$. Episodic social cognition was higher in younger participants $(b=0.10,95 \% \mathrm{Cl}[0.02-0.18], t(193)=2.47, p=.014)$. There was also a significant interaction between Sample (pre- vs during-lockdown) and Social environment $(F(2)=6.06, p=$ .002). This interaction indicated that although episodic social cognition was most prevalent when interacting with others in both samples, the increase in episodic social cognition between 'interacting' with both 'alone' (unadjusted, $b=0.25,95 \% \mathrm{CI}[0.11,0.39], \mathrm{t}(4844)=3.44, \mathrm{p}<.001$ ) and 'not interacting' (unadjusted, $b=0.17,95 \% \mathrm{CI}[0.01,0.34], \mathrm{t}(4795)=2.03, \mathrm{p}=.042$ ) was greater in the lockdown sample. During lockdown, therefore, although social interactions were less frequent, when they did occur, they were associated with greater evidence of episodic social cognition. 
Model 4: Imagery. There was a significant three-way interaction between Sample, Age-group, and Social environment $(F(2)=5.85, p=.003)$. Pairwise comparisons at each level of Social environment, split by Sample and Age-group (Bonferroni adjusted for 12 tests) revealed that for younger participants, the direction of the effect of Social environment on levels of imagery differed between samples. Pre-lockdown, younger participants reported less imagery when they were alone compared to when they were interacting with others $(b=-0.14,95 \% \mathrm{CI}[-0.27,-0.01], t(4745)$ $=-2.98, p=.035$ ) and during-lockdown, younger participants reported more imagery when they were alone compared to when they were interacting with others $(b=0.20,95 \% \mathrm{Cl}[0.01,0.38]$, $t(4855)=3.05, p=.028)$. A comparison of these contrasts confirmed that this difference was significant (unadjusted, $b=-0.33,95 \% \mathrm{Cl}[-0.49,-0.18], t(4845)=-4.21, p<.001$ ). Therefore, during lockdown, younger participants reported more imagery when they were alone compared to when interacting with others.

Model 5: Detailed task focus. There were no significant main effects or interactions ( $p$ 's $>.05$ ). Therefore, the lockdown had no significant impact on the overall prevalence of detailed task focus.

250 Comparing thought patterns between (i) current activities and (ii) age groups during 251 lockdown.

252 To understand how changes to people's daily routine, including changes to working, influenced 253 patterns of ongoing thought during lockdown, we next explored the links between ongoing thought patterns and ongoing activities. In the baseline sample, we had not obtained information about concurrent activities; however, in the lockdown sample, we asked participants to describe the primary activity they were performing (see Methods). The twenty-four options were condensed into five categories for analysis: (i) Working, (ii) Leisure activities, (iii) Social interactions, (iv) Media consumption and (v) Essential tasks (see Supplementary Information). We conducted a series of models examining whether patterns of thought varied significantly between activity categories and whether there were age-related differences (see Methods). As before, the alpha level was set to $<.01$ (two-tailed) to account for family-wise error emerging from conducting five models. These results are summarized in Figure 3 (see Tables S7-S9 for ANOVA output, parameter estimates, and the variance explained by random effects).

Model 1: Future-directed problem-solving. There was a significant main effect of Activity $(F(4)$ $=33.67, p<.001)$. Future-directed problem-solving was higher when participants were working during lockdown $(b=0.62,95 \% \mathrm{Cl}[0.48,0.77], t(1689)=8.62, p<.001)$ and lower when consuming media $(b=-0.36,95 \% \mathrm{Cl}[-0.44,-0.28], t(1743)=-8.92, p<.001)$ or engaging in essential tasks $(b=-0.22,95 \% \mathrm{Cl}[-0.30,-0.14], t(1727)=-5.59, p<.001)$. Therefore, while future-directed problem-solving was significantly lower in the lockdown sample, this pattern of thought was reinstated when individuals engaged in work.

Model 2: Pleasant engagement. There was a significant main effect of Activity $(F(4)=18.93, p<$ .001). Pleasant engagement was higher during leisure activities $(b=0.27,95 \% \mathrm{Cl}[0.19,0.36]$, $t(1712)=6.36, p<.001)$ and lower when participants consumed media $(b=-0.21,95 \% \mathrm{Cl}[-0.29$, -0.13], $t(1730)=-5.14, p<.001)$ or during social interactions $(b=-0.12,95 \% \mathrm{Cl}[-0.23,-0.00]$, $t(1719)=-2.03, p=.043)$. There was also a significant interaction between Activity and Agegroup $(F(4)=5.71, p<.001)$. Pairwise comparisons at each level of Age-group, split by Activity (Bonferroni adjusted for 5 tests) revealed that pleasant engagement was higher for older participants when working compared to younger participants $(b=0.78,95 \% \mathrm{Cl}[0.19,1.37], t(350)$ $=3.43, p=.003)$.

Model 3: Episodic social cognition. There was a significant main effect of Activity $(F(4)=$ 282 $25.58, p<.001)$. Episodic social cognition was higher during social interactions $(b=0.52,95 \% \mathrm{Cl}$ $[0.40,0.64], t(1738)=8.72, p<.001)$ and lower when consuming media $(b=-0.23,95 \% \mathrm{Cl}[-$ 
$0.31,-0.14], t(1752)=-5.42, p<.001)$ or working $(b=-0.35,95 \% \mathrm{Cl}[-0.50,-0.20], t(1696)=-$ $2844.68, p<.001)$. Mas a significant main effect of Activity $(F(4)=6.52, \rho<.001)$. Imagery was higher when participants consumed media $(b=0.17,95 \% \mathrm{Cl}[0.09,0.26], t(1719)=4.21, p$ $<.001)$ and lower when engaging in essential tasks $(b=-0.09,95 \% \mathrm{Cl}[-0.17,-0.01], t(1700)=-$ $2.30, p=.022)$.

Model 5: Detailed task focus. There was a significant main effect of Activity $(F(4)=13.38, p<$ $.001)$. Detailed task focus was higher when working $(b=0.39,95 \% \mathrm{Cl}[0.25,0.53], t(1656)=$ $5.44, p<.001)$ or during social interactions $(b=0.13,95 \% \mathrm{Cl}[0.02,0.24], t(1730)=2.30, p=$ $.021)$ and lower when engaging in essential tasks $(b=-0.16,95 \% \mathrm{Cl}[-0.24,-0.09], t(1733)=-$ $4.13, p<.001)$, leisure activities $(b=-0.19,95 \% \mathrm{Cl}[-0.27,-0.11], t(1731)=-4.48, p<.001)$ or when consuming media $(b=-0.17,95 \% \mathrm{Cl}[-0.25,-0.09], t(1741)=-4.23, p<.001)$. There was a significant interaction between Activity and Age-group $(F(4)=5.04, p<.001)$. Pairwise comparisons at each level of Age-group, split by Activity (Bonferroni adjusted for 5 tests) revealed that detailed task focus was higher when older participants engaged in social interactions compared to younger participants $(b=0.48,95 \% \mathrm{Cl}[0.02,0.93], t(308)=2.72, p=.034)$.

\section{Comparing thought patterns between (i) virtual and physical social interactions and (ii) age groups during lockdown.}

During lockdown, while people were unable to socialize in person with people outside of their household, they could still interact virtually. In the baseline group, we did not collect information regarding whether social interactions were virtual. However, in the lockdown sample, participants reported on both their physical and virtual interactions. To examine the effects of virtual social interaction on thoughts in the lockdown sample, we conducted a series of models in which each thought pattern was the outcome measure, and Interaction type and Age-group were the explanatory variables (see Supplementary Information). Interaction type had four levels: (i) no interaction at all, (ii) virtual interaction only, (iii) physical interaction only and (iv) both virtual and physical interaction (see Table S10 for how this variable was coded). As before, the alpha level was set to <.01 (two-tailed) to account for family-wise error emerging from conducting five models. These results are summarized in Fig. S3 and see Tables S11-S13 for ANOVA output, parameter estimates, and variance explained by random effects.

We found that future-directed problem-solving was less apparent when participants were physically- compared to virtually- interacting, while episodic social cognition was more apparent across all forms of interaction when compared to not interacting at all. In addition, although the effects did not pass the Bonferroni correction, patterns of imagery were less apparent when physically interacting compared to virtually interacting, particularly for younger participants. Finally, detailed task focus was more apparent when virtually interacting compared to when interacting both virtually and physically, and not interacting at all. Notably, for older participants, detailed task focus was more apparent during virtual interactions compared to all other forms of interaction and when not interacting at all. However, it is worth noting that the cells of this analysis were unbalanced, with fewer observations for interacting- particularly virtually- compared to not interacting at all (see Table S14 for number of observations per factor level by Age-group), so these results should be interpreted with caution.

\section{Relationship to affect}

Finally, we conducted an exploratory analysis to understand whether the lockdown-related changes in ongoing thought identified in our prior analysis were independent of changes in affect (see Supplementary Information). Importantly, including affect did not substantially change the lockdown-related results reported above. However, the main effects of Age-group for models 1-3 no longer reached significance (see Supplementary Information). In addition, we ran a parallel 
analysis in which we compared the prevalence of negative and positive affect between Samples, Social environments, and Age-groups to examine how state affect may have changed during lockdown (see Supplementary Information for further details).

\section{Discussion}

Our study set out to determine how specific features of the UK's first lockdown corresponded with changes in ongoing thought patterns in daily life, focusing on changes to socializing and working. The contents of ongoing thoughts were assessed using multidimensional experience-sampling (MDES) (37), an established method with documented neural (e.g., 40, 41, 43, 46) and behavioral correlates (e.g., 28, 47). Our analysis identified five thought patterns: future-directed problem-solving, pleasant engagement, episodic social cognition, imagery, and detailed task focus. Importantly, these five thought patterns are consistent with previous research using this method $(15,17,40,41,45,46)$.

One goal of our study was to assess how changes in socialization during lockdown impacted patterns of social thought in daily life. Across both samples, in-person social interaction was associated with increased episodic social cognition, reduced future-directed problem-solving and greater pleasant engagement in younger individuals. During lockdown, opportunities for social interactions were reduced, but when social interactions did occur, episodic social cognition was especially prevalent. So, although participants were less able to engage in in-person social interactions during lockdown, when those interactions were possible, they promoted greater increases in social thinking than would normally occur. Furthermore, during lockdown, all types of interaction- both virtual and in-person- were associated with increased episodic social cognition suggesting that online interactions may partly ameliorate the consequences of lockdown on social cognition. Importantly, since the lockdown was a natural experiment in how changes in socialization affect our thinking in daily life, our findings provide real-world confirmation of laboratory evidence linking social thinking to the availability of social interactions (11) and are consistent with the possibility that ongoing thought helps facilitate interactions either in the moment or in the future $(11,48)$. Our study, therefore, provides ecologically valid evidence to support theoretical perspectives that highlight how social interactions shape social thought patterns in daily life $(11,12)$.

The second goal of our study was to understand how changes in opportunities for working during lockdown influenced ongoing thought patterns in daily life. Future-directed problem-solving, something generally prevalent in younger individuals, was (i) significantly reduced during lockdown relative to pre-lockdown but (ii) was highest during lockdown when individuals were working. Our results, therefore, suggest that when external commitments are disrupted (in this case, via lockdown), future-directed problem-solving is reduced unless people are working. Thus, our data support theories suggesting that the "prospective bias" in ongoing thought is related to goal-related processes since it was disrupted by lockdown unless people were actively engaged in work $(18,25-28,49)$. Moreover, given research showing goal-directed planning is reduced in dysphoric individuals (50) and that future thinking is important for maintaining mental health (24, 29,30 ), our study raises the possibility that reduced opportunities for work may contribute to the negative emotional changes documented during lockdown $(4,5,10)$ via a reduction in futurerelated thinking- an important question for future work to explore.

Our final goal was to understand whether lockdown differentially impacted thinking patterns in older and young individuals. Consistent with prior research (31-33), we found evidence for age differences in ongoing thought patterns. For example, younger individuals reported higher levels of future-directed problem-solving and episodic social cognition, and lower levels of pleasant engagement during activities than older adults. We also found that before lockdown, younger individuals reported more imagery when interacting with others, whereas during lockdown, imagery was higher when younger individuals were alone. This thought pattern was associated with media consumption during lockdown, so it is plausible that this increased imagery in younger 
adults when alone was related to an increase in media usage (51). Finally, for older participants, virtual interactions during lockdown were linked to increased detailed task focus, a pattern that might reflect the effort required when interacting online, possibly capturing the phenomenon of 'zoom fatigue' (52).

In summary, the restrictions introduced during the UK's first national lockdown brought reduced opportunities for socialization and working. In parallel with these changes in daily routine, we found changes in the patterns of thinking associated with these activities. Specifically, during lockdown, social interactions promoted a greater increase in episodic social thinking than prelockdown, and while future-directed problem-solving was significantly reduced during lockdown, this thought pattern increased when individuals engaged in work. Therefore, on the limited occasions that individuals were able to socialize or work during lockdown, these activities had a significant effect on relevant thought patterns, highlighting the important role that our daily routine has on our thinking.

Although our study sheds light on how lockdown changed ongoing thought patterns in daily life, several limitations should be considered when interpreting these results. First, our study capitalized on an existing dataset to provide a baseline to understand how thought patterns changed during lockdown. While this design feature was unavoidable given the pandemic's unforeseen nature, conclusions regarding the impact of lockdown would have been stronger if we could have examined within-person changes in the same participants over time. Importantly, however, we established that the underlying structure of ongoing thought was almost identical in both samples (see Fig. S2), supporting the validity of the pre-lockdown sample as a baseline. Future work should aim to track people's thoughts in the moment longitudinally, through periods of lockdown and during periods of lockdown relaxation. Second, our analyses of the relationship between current activities (e.g., working) and ongoing thought are based only on the lockdown sample. Therefore, while our data allow the determination of how changes in working opportunities contributed to cognition during lockdown, it is unclear how working influences thought patterns in a more normal context. Finally, it is important to note that there are other influences on people's ongoing thoughts during lockdown, beyond those assessed in our study. For example, the current study did not account for economic changes, fear of illness, whether an individual (or close friend/family member) contracted COVID-19 during the study or bereavements. Nonetheless, our study suggests that in addition to other changes in life circumstances, changes to socialization and opportunities for work are important contributors to how lockdowns influence the contents of people's thoughts in daily life.

Our examination of how broad, naturally occurring changes in society influence cognition also raises important questions for future investigations of ongoing thought. Emerging evidence highlights the lockdown's consequences on mental health $(4,5,10)$ so future studies should examine relationships between risk factors such as anxiety and depression and ongoing thought in daily life and during lockdowns. Furthermore, our data indicate that both younger and older adults reported being alone more in the lockdown sample than pre-lockdown. However, we could not make an equivalent comparison of changes in specific daily activities (including work). Therefore, it remains unclear the extent to which different daily routines in younger and older adults may contribute to age differences in thought patterns. Finally, although studies conducted before the pandemic show that features of ongoing thoughts (e.g., a focus on the future) are prevalent across cultures (14), our study used a UK sample, so it is important to understand how lockdowns change ongoing thought patterns across cultures.

We close by considering the implications of our study for understanding ongoing thought patterns in daily life. Prior studies investigating ongoing thought have focussed on assessing thought within laboratory and neuroimaging contexts, revealing links between thought content and neural activity (e.g., 37, 41, 43, 47, 53), cognitive ability (e.g., 54, 55), affective style (e.g., 15, 56, 57), as well as task and social contexts $(11,15)$. Our study complements these findings by highlighting 
the role that aspects of our daily routines- particularly social interactions and work- play in shaping our cognition. It is perhaps unsurprising that ongoing thought patterns are shaped by these activities since (i) we spend a large proportion of our lives interacting with others (11) and working, and (ii) that successful adaptation within both of these domains is critical for wellbeing. For example, loneliness increases the likelihood of death by $26 \%$ (58), while unemployment is associated with reduced psychological and physical wellbeing (59). In this way, our study illustrates that features of a person's daily routine are important in scaffolding their ongoing thought patterns, and highlights that experience-sampling in naturalistic contexts is an important way to understand when and how what we do influences ongoing human cognition, both during lockdowns and in more normal times.

\section{Materials and Methods Participants}

The full study protocol was approved by the Psychology Department ethics committee at the University of York. All participants gave informed consent (either written or online) before taking part and were debriefed upon completion. In the pre-lockdown sample, younger participants were recruited between October 2016 and March 2017 from undergraduate and postgraduate student bodies and were either paid or given course credits. Seventy-eight younger participants completed experience-sampling surveys (female $=57$, male $=21$, Age: $M=19.64, S D=1.62$, range $=18-27$ ). These data have been analyzed and reported previously by Ho et al. (17). In the pre-lockdown sample, older participants were recruited between August 2016 and November 2016 and were paid for their time. Thirty-five older participants completed experience-sampling surveys (female $=20$, male $=15$, Age: Mean $=66.80, S D=6.88$, range $=55-87$ ). In the lockdown sample, all participants were invited to participate in the daily-life experience-sampling after completing an initial survey, as part of a larger project, on Prolific (www.prolific.co). All participants were paid for their time. Ninety-one participants completed experience-sampling surveys between April $29^{\text {th }}, 2020$, and May $13^{\text {th }}, 2020$. Two participants were removed from the study on day one as they were not currently residing in the UK, and their data were excluded. Two participants were excluded for having missing age data. Five participants were excluded as they did not fall into either the young (18-35 years) or older (55+ years) age-groups. The final sample comprised fifty-nine younger participants (female $=40$, male $=17$, self-describe $=1$, prefer not to say= 1, Age: Mean: $24.22, \mathrm{SD}=4.07$, range $=18-35$ ) and twenty-three older participants (female $=13$, male $=9$, self-describe $=1$, Age: Mean: $63.91, \mathrm{SD}=7.06$, range $=55-78$ ) .

\section{Procedure}

Participants received a text message with a link to an online Qualtrics survey five times daily for seven days at quasi-random intervals between 09:00 and 21:00 (20:45 in the lockdown sample) administered via SurveySignal (60). Each survey link expired after two hours. In the pre-lockdown sample, seven older participants completed up to eight surveys a day for ten days. However, this procedure was shortened after participant feedback that the procedure was too intensive. Rerunning our analyses removing these additional observations did not substantially change the results. Additionally, in the pre-lockdown sample, twenty-three older participants and one younger participant opted to complete the study on paper. They were provided with a phone where texts acted as signals (see Supplementary Information for comparison of completion type). Participants in both samples also completed daily diary questionnaires, and participants in the lockdown sample completed an exit questionnaire at the end of the study. These questionnaires did not sample ongoing thought and are therefore not reported here.

\section{Experience-sampling surveys.}

The experience-sampling survey first asked participants to consider the contents and form of their thoughts immediately before being signalled on various dimensions using a 1-5 Likert scale. We sought to compare thought patterns observed across both samples, so we focused on the twentytwo items present in both (see Table S1). The survey then asked participants to rate their 
emotions and feelings on various dimensions using a 1-5 Likert scale (see Table S15 for the 12 affect items present in both samples that were included in supplementary analyses). Participants were also asked "Were you alone or with other people just before taking this survey?" (in the lockdown sample, the question specified 'physically and not virtually'). Response options were: "Alone", "Around people but NOT interacting" or "Around people and interacting". In the lockdown sample, participants were also asked "Virtually, were you alone or with other people just before taking this survey?". Response options were the same as those for the physical interaction question. Additionally, in the lockdown sample, participants were asked to indicate their location (seven options; see Figure 1 panel B) and primary activity (twenty-four options; see Supplementary Information) immediately before answering the survey. The activity options were based on those used in the 'Day reconstruction method' (61) and modified to include activities that were likely to be prevalent during lockdown. In both samples, participants were also asked several other questions about their ongoing experience (e.g., whether they had recently accessed new information regarding COVID-19), which are not the focus of this paper. All experiencesampling survey questions and response options included in the current study are available in the Supplementary Information (Tables S1, S15, S23).

\section{Analysis}

\section{Data and code availability statement}

For details of $\mathrm{R}$ packages used in analysis, see Supplementary Information. All code used in analysis and preparation of figures is available online at https://github.com/BronteMckeown/pre vs during lockdown ESQ analysis. All anonymized data used in the preparation of this manuscript is openly available via Mendeley data (http://dx.doi.org/10.17632/xv6dv2drm8.1).

\section{Assessing changes to daily-life during lockdown}

To assess whether the percentage of responses for which participants reported being alone was higher in the lockdown sample than the pre-lockdown sample, we first calculated the percentage of each participant's responses in which they said they were (i) alone, (ii) around people but not interacting with them and (iii) around people and interacting with them. We then ran a two-way ANOVA with each participant's 'alone' percentage as the outcome variable and Sample (pre- vs during-lockdown) and Age-group (young vs older) as the predictors. To examine where participants were located in the lockdown sample, we calculated the overall percentage of responses for each 'location' option.

\section{Preparing data for PCA}

Two experience-sampling questions ('Positive' and 'Deliberate') in the pre-lockdown sample were measured on 7, rather than 5-point scales. All questions were therefore rescaled using the following computation: (observed score-1)/(highest possible score on that scale-1). The rescaled questions were then z-scored before applying Principal Components Analysis (PCA) to the combined data.

\section{PCA}

To identify common "patterns of thought" between both samples, PCA with varimax rotation was applied to the combined thought data from both samples (twenty-two items; see Table S1) using IBM SPSS Statistics (Version 26). PCA was applied at the observation level in the same manner as in our previous studies (e.g., 15, 24, 43). The Kaiser-Meyer-Olkin measure of sampling adequacy was .84 , above the commonly recommended value of .6 , and Bartlett's test of sphericity was significant $(x 2(231)=28737.22, p<.001)$. Five components, with an eigenvalue $>1$, were retained for inclusion as outcome variables in the linear mixed models. To ensure that 
the thought patterns identified across samples were present in both samples separately, we ran a PCA on each sample separately (specified five components for extraction) and correlated each participants PCA score from this analysis with their PCA score from the combined analysis (see Fig. S2 for scatterplots).

\section{$542 \quad$ Linear mixed models}

543 Linear mixed models (LMMs) were fitted by restricted maximum-likelihood estimation in R (4.0.2 544 (62)) using the Ime4 package (1.1.26 (63)). We used the ImerTest package (3.1.3 (64)) to obtain $\mathrm{p}$-values for the $\mathrm{F}$ and $\mathrm{t}$-tests returned by the Ime4 package. For each set of models, the alpha level was set based on .05 divided by the number of models (i.e., Bonferroni corrected alpha level). Degrees of freedom were calculated using Satterthwaite approximation. For F-tests, Type 3 Sum of Squares was chosen because imbalances in the data are assumed to occur randomly and not due to differences in the population (65). Contrasts were set to 'contr.sum', meaning that the intercept of each model corresponds to the grand mean of all conditions and that when a factor has two levels, the parameter estimate is equal to half of the difference between the two levels (65). Estimated marginal means were calculated using the emmeans package (1.5.3 (66)). Post-hoc pairwise comparisons were also calculated using the emmeans package (66) and corrected for multiple comparisons using the 'Bonferroni' adjustment, which adjusts both the confidence intervals and $p$-values associated with each estimate and test. For contrasts of contrasts, custom contrasts were set manually, so could not be adjusted for multiple comparisons. Across all models, to account for multiple observations per participant, day number was nested within participant as a random intercept.

\section{Comparing thought patterns between (i) pre- and during-lockdown samples, (ii) age- groups and (iii) social environments}

We ran five LMMs- one with each thought component as the outcome variable modelling the following fixed factors and their interactions: (i) 'Sample' (2 levels: pre- and during-lockdown), (ii) 'Age-group' (2 levels: younger and older) and (iii) 'Social environment' (3 levels: alone, around people but not interacting, around people and interacting). Age-group mean-centered age was included in all models as a nuisance covariate to control for age differences, within age-groups, between the two samples. In total, 195 participants (4870 observations) were included in these models.

Example model formula: Imer(Thought component $x \sim$ Sample * Age-group * Social environment + Age-group mean centered age + (1|Participant/Day number $)$

In addition, to account for differences in age-range in the younger groups between pre- and during-lockdown samples, we re-ran these analyses while limiting the age-range for the younger group to 18-27 years in both samples. Re-running our analyses in this way did not change the overall interpretations of the paper (see Supplementary Information).

\section{Comparing thought patterns between (i) activities and (ii) age-groups in the lockdown sample}

We ran five LMMs- one with each thought component as the outcome variable modelling the following fixed factors and their interactions: (i) 'Activity' (5 levels) and (ii) 'Age-group' (2 levels). The 'activity' question had twenty-four options which we condensed for analyses. Any observations containing the option 'Other' $(\mathrm{N}=88)$ were removed, leaving 81 participants (1777 observations) in the model. The remaining options were grouped into five categories (i) Working, (ii) Leisure activities, (iii) Social interactions, (iv) Media consumption and (v) Essential tasks (see Supplementary Information for details). 
583 Example model formula: Imer(Thought component $x \sim$ Activity * Age-group + (1|Participant/Day 584 number)

\section{Acknowledgments}

This project was supported by European Research Council Consolidator awarded to JS [WANDERINGMINDS-646927] and an award from the Dunhill Medical trust to JS, LR and EJ [R422/0515].

\section{References}

1. B. Chiripanhura, B. Carrera, E. Monahan, Furloughing of workers across UK business: 23 March 2020 to 5 April 2020. Office for National Statistics, available at https://www.ons.gov.uk/employmentandlabourmarket/peopleinwork/employmentandemployeetypes larticles/furloughingofworkersacrossukbusinesses/latest (accessed 21 July, 2020) (2020).

2. M. Stephens, S. Cross, G. Luckwell, Coronavirus and the impact on output in the UK economy: June 2020. Office for National Statistics 12 (2020).

3. A. Campbell, S. Caul (2020) Deaths involving COVID-19, England and Wales: deaths occurring in May 2020. Office for National Statistics, available at https://backup.ons.gov.uk/wpcontent/uploads/sites/3/2020/06/Deaths-involving-COVID-19-England-and-Wales-deaths-occurringin-May-2020.pdf (accessed 17 June, 2021) (2020).

4. R. G. White, C. Van Der Boor, Impact of the COVID-19 pandemic and initial period of lockdown on the mental health and well-being of adults in the UK. BJPsych open 6 (2020).

5. J. Banks, X. Xu, The mental health effects of the first two months of lockdown and social distancing during the Covid-19 pandemic in the UK. IFS Working Papers No. W20/16 (2020).

6. J. Huckins et al., Mental Health and Behavior During the Early Phases of the COVID-19 Pandemic: A Longitudinal Mobile Smartphone and Ecological Momentary Assessment Study in College Students. Journal of medical Internet research, 22(6), e20185 (2020).

7. E. Robinson et al., Obesity, eating behavior and physical activity during COVID-19 lockdown: A study of UK adults. Appetite, 104853 (2020).

8. O. Giuntella, K. Hyde, S. Saccardo, S. Sadoff, Lifestyle and mental health disruptions during Covid19. Proceedings of the National Academy of Sciences 118 (2021).

9. J. M. Groarke et al., Loneliness in the UK during the COVID-19 pandemic: Cross-sectional results from the COVID-19 Psychological Wellbeing Study. PloS one 15, e0239698 (2020).

10. R. C. O'Connor et al., Mental health and well-being during the COVID-19 pandemic: longitudinal analyses of adults in the UK COVID-19 Mental Health \& Wellbeing study. The British Journal of Psychiatry, 1-8 (2020).

11. J. Mildner, D. Tamir, The people around you are inside your head: Social context shapes spontaneous thought. PsyArXiv [Preprint] (2018). https://doi.org/10.31234/osf.io/xmzh7 (accessed 17 June, 2020).

12. G. Poerio, J. Smallwood, Daydreaming to navigate the social world: What we know, what we don't know, and why it matters. Social and Personality Psychology Compass 10, 605-618 (2016).

13. R. A. Mar, M. F. Mason, A. Litvack, How daydreaming relates to life satisfaction, loneliness, and social support: the importance of gender and daydream content. Consciousness and cognition 21, 401-407 (2012).

14. X. Song, X. Wang, Mind wandering in Chinese daily lives-an experience sampling study. PloS one 7, e44423 (2012).

15. D. Konu et al., Exploring patterns of ongoing thought under naturalistic and conventional taskbased conditions. Consciousness and cognition 93, 103139 (2021).

16. G. Poerio, P. Totterdell, L.-M. Emerson, E. Miles, Social daydreaming and adjustment: an experience-sampling study of socio-emotional adaptation during a life transition. Frontiers in psychology 7, 13 (2016).

17. N. S. P. Ho et al., Facing up to why the wandering mind: Patterns of off-task laboratory thought are associated with stronger neural recruitment of right fusiform cortex while processing facial stimuli. Neurolmage, 116765 (2020).

18. B. Baird, J. Smallwood, J. W. Schooler, Back to the future: autobiographical planning and the functionality of mind-wandering. Consciousness and cognition 20, 1604-1611 (2011).

19. S. R. Gold, J. P. Reilly III, Daydreaming, current concerns and personality. Imagination, Cognition and Personality 5, 117-125 (1985). 
$64020 . \quad$ E. Klinger, S. G. Barta, M. E. Maxeiner, Motivational correlates of thought content frequency and

21. D. Stawarczyk, S. Majerus, M. Maj, M. Van der Linden, A. D'Argembeau, Mind-wandering: Phenomenology and function as assessed with a novel experience sampling method. Acta psychologica 136, 370-381 (2011).

22. J. S. Antrobus, J. L. Singer, S. Greenberg, Studies in the stream of consciousness: experimental enhancement and suppression of spontaneous cognitive processes. Perceptual and Motor Skills 23, 399-417 (1966).

23. H. B. Kappes, B. Schwörer, G. Oettingen, Needs instigate positive fantasies of idealized futures. European Journal of Social Psychology 42, 299-307 (2012).

24. F. J. Ruby, J. Smallwood, H. Engen, T. Singer, How self-generated thought shapes mood-the relation between mind-wandering and mood depends on the socio-temporal content of thoughts. PloS one 8, e77554 (2013).

25. D. Stawarczyk, H. Cassol, A. D'Argembeau, Phenomenology of future-oriented mind-wandering episodes. Frontiers in Psychology 4, 425 (2013).

26. A. D'Argembeau, O. Renaud, M. Van der Linden, Frequency, characteristics and functions of future-oriented thoughts in daily life. Applied Cognitive Psychology 25, 96-103 (2011).

27. E. Klinger, E. H. Koster, I. Marchetti, Spontaneous thought and goal pursuit: From functions such as planning to dysfunctions such as rumination. The Oxford handbook of spontaneous thought: Mind-wandering, creativity, and dreaming, 215 (2018).

28. B. Medea et al., How do we decide what to do? Resting-state connectivity patterns and components of self-generated thought linked to the development of more concrete personal goals. Experimental brain research 236, 2469-2481 (2018).

29. E. C. Hunter, R. C. O'Connor, Hopelessness and future thinking in parasuicide: The role of perfectionism. British Journal of Clinical Psychology 42, 355-365 (2003).

30. R. O'Connor, D. O'Connor, S. O'Connor, J. Smallwood, J. Miles, Hopelessness, stress, and perfectionism: The moderating effects of future thinking. Cognition \& Emotion 18, 1099-1120 (2004).

31. M. Irish, Z.-I. Goldberg, S. Alaeddin, C. O'Callaghan, J. R. Andrews-Hanna, Age-related changes in the temporal focus and self-referential content of spontaneous cognition during periods of low cognitive demand. Psychological research 83, 747-760 (2019).

32. D. Maillet et al., Aging and the wandering brain: Age-related differences in the neural correlates of stimulus-independent thoughts. PloS one 14, e0223981 (2019).

33. D. Maillet et al., Age-related differences in mind-wandering in daily life. Psychology and aging 33 , 643 (2018).

34. L. M. Giambra, Daydreaming across the life span: Late adolescent to senior citizen. The International Journal of Aging and Human Development 5, 115-140 (1974).

35. R. Zhaoyang, M. J. Sliwinski, L. M. Martire, J. M. Smyth, Age differences in adults' daily social interactions: An ecological momentary assessment study. Psychology and aging 33, 607 (2018).

36. R. Larson, M. Csikszentmihalyi, "The experience sampling method" in Flow and the foundations of positive psychology. (Springer, 2014), pp. 21-34.

37. J. Smallwood et al., Representing representation: Integration between the temporal lobe and the posterior cingulate influences the content and form of spontaneous thought. PloS one 11, e0152272 (2016).

38. L. M. Martinon, J. Smallwood, D. McGann, C. Hamilton, L. M. Riby, The disentanglement of the neural and experiential complexity of self-generated thoughts: A users guide to combining experience sampling with neuroimaging data. Neurolmage 192, 15-25 (2019).

39. J. Smallwood et al., The neural correlates of ongoing conscious thought. iScience, 102132 (2021).

40. T. Karapanagiotidis et al., The psychological correlates of distinct neural states occurring during wakeful rest. Scientific reports 10, 1-11 (2020).

41. D. Konu et al., A role for the ventromedial prefrontal cortex in self-generated episodic social cognition. Neurolmage 218, 116977 (2020).

42. A. Turnbull et al., Left dorsolateral prefrontal cortex supports context-dependent prioritisation of offtask thought. Nature communications 10, 1-10 (2019).

43. H.-T. Wang et al., Dimensions of experience: exploring the heterogeneity of the wandering mind. Psychological science 29, 56-71 (2018).

44. T. Karapanagiotidis, E. Jefferies, J. Smallwood, Interactions between the neural correlates of dispositional internally directed thought and visual imagery. Philosophical Transactions of the Royal Society B 376, 20190691 (2021). 
45. A. Turnbull et al., The ebb and flow of attention: Between-subject variation in intrinsic connectivity and cognition associated with the dynamics of ongoing experience. Neuroimage 185, 286-299 (2019).

46. M. Sormaz et al., Default mode network can support the level of detail in experience during active task states. Proceedings of the National Academy of Sciences 115, 9318-9323 (2018).

47. A. Turnbull et al., Reductions in task positive neural systems occur with the passage of time and are associated with changes in ongoing thought. Scientific reports 10, 1-10 (2020).

48. M. L. Meyer, Social by default: characterizing the social functions of the resting brain. Current directions in psychological science 28, 380-386 (2019).

49. L. Kvavilashvili, J. Rummel, On the nature of everyday prospection: A review and theoretical integration of research on mind-wandering, future thinking, and prospective memory. Review of General Psychology 24, 210-237 (2020).

50. B. Plimpton, P. Patel, L. Kvavilashvili, Role of triggers and dysphoria in mind-wandering about past, present and future: A laboratory study. Consciousness and Cognition 33, 261-276 (2015).

51. G. Vassilev, A "new normal"? How people spent their time after the March 2020 coronavirus lockdown - Office for National Statistics, available at https://www.ons.gov.uk/peoplepopulationandcommunity/healthandsocialcare/conditionsanddisease s/articles/anewnormalhowpeoplespenttheirtimeafterthemarch2020coronaviruslockdown/2020-12-09 (accessed 23 June, 2021) (2020).

52. S. Cranford, Zoom Fatigue, Hyperfocus, and Entropy of Thought. Matter 3, 587-589 (2020).

53. B. Mckeown et al., The relationship between individual variation in macroscale functional gradients and distinct aspects of ongoing thought. Neurolmage 220, 117072 (2020).

54. M. J. Kane et al., For whom the mind wanders, and when: An experience-sampling study of working memory and executive control in daily life. Psychological science 18, 614-621 (2007).

55. J. Rummel, C. D. Boywitt, Controlling the stream of thought: Working memory capacity predicts adjustment of mind-wandering to situational demands. Psychonomic bulletin \& review 21, 13091315 (2014).

56. Y.-Q. Deng, S. Li, Y.-Y. Tang, The relationship between wandering mind, depression and mindfulness. Mindfulness 5, 124-128 (2014).

57. F. Hoffmann, C. Banzhaf, P. Kanske, F. Bermpohl, T. Singer, Where the depressed mind wanders: Self-generated thought patterns as assessed through experience sampling as a state marker of depression. Journal of affective disorders 198, 127-134 (2016).

58. J. Holt-Lunstad, T. B. Smith, M. Baker, T. Harris, D. Stephenson, Loneliness and social isolation as risk factors for mortality: a meta-analytic review. Perspectives on psychological science 10, 227237 (2015).

59. F. McKee-Ryan, Z. Song, C. R. Wanberg, A. J. Kinicki, Psychological and physical well-being during unemployment: a meta-analytic study. Journal of applied psychology 90, 53 (2005).

60. W. Hofmann, P. V. Patel, SurveySignal: A convenient solution for experience sampling research using participants' own smartphones. Social Science Computer Review 33, 235-253 (2015).

61. D. Kahneman, A. B. Krueger, D. A. Schkade, N. Schwarz, A. A. Stone, A survey method for characterizing daily life experience: The day reconstruction method. Science 306, 1776-1780 (2004).

62. R Core Team, R: A Language and Environment for Statistical Computing, R Foundation for Statistical Computing, Vienna, Austria. https://www.r-project.org/ (2020).

63. D. Bates, M. Maechler, B. Bolker, S. Walker, Fitting Linear Mixed-Effects Models Using Ime4, Journal of Statistical Software 67, 1-48 https://doi.org/10.18637/jss.v067.i01 (2015).

64. A. Kuznetsova, P. B. Brockhoff, R. H. Christensen, ImerTest Package: Tests in Linear Mixed Effects Models. Journal of statistical software 82(13), 1-26 https://doi.org/10.18637/jss.v082.i13 (2017).

65. H. Singmann, D. Kellen, An introduction to mixed models for experimental psychology. New methods in cognitive psychology 28, 4-31 (2019).

66. R. Lenth, Emmeans: Estimated marginal means, aka least-squares means. R package version 1.5.3. https://CRAN.R-project.org/package=emmeans (2020).

\section{Figures Legends}

Figure 1. Changes to daily life during lockdown and patterns of ongoing thought identified across both experience-sampling datasets (pre- and during-lockdown). (A) Bar chart comparing the 
mean percentage of experience-sampling responses in which participants said they were (i) alone, (ii) around other people but not interacting and (iii) around people and interacting, between age-groups and samples- demonstrating that during-lockdown, both age-groups reported being alone more than pre-lockdown. Error bars represent $95 \%$ confidence intervals ( $\mathrm{N}$ observations = 4955). (B) Pie chart shows the percentage of responses for each location option in the lockdown sample, demonstrating that the majority (85\%) of responses were 'Inside at home' ( $N$ observations = 1865). (C) Word clouds representing the item loadings on the five "patterns of thought" identified in the thought data from both samples (pre- and during-lockdown) ( $\mathrm{N}$ observations $=4876$ ) using Principal Components Analysis. Each word represents an experience-sampling item (twenty-two items; see Table S1). Font size represents the magnitude of the loading, and the color describes the direction. Warm colors reflect positive loadings, while cool colors reflect negative loadings (see Table S2 for exact component loadings).

769

770

771

772

Figure 2. A summary of the linear mixed models' results comparing the prevalence of each thought pattern between (i) pre- and during-lockdown samples, (ii) age-groups and (iii) social environments. On the left-hand side, there are the word clouds representing each thought pattern. Each word represents an experience-sampling item (see Table S1). Font size represents the magnitude of the loading, and the color describes the direction. Warm colors reflect positive loadings, while cool colors reflect negative loadings. The y-axis of each graph shows the predicted means for each thought pattern. The x-axis shows the social environment options: (i) alone, (ii) around people but not interacting and (iii) around people and interacting. White bars represent the pre-lockdown sample, and grey bars represent the lockdown sample. Each bar graph is split by age-group, with young participants on the left and older on the right. Error bars represent the $95 \%$ confidence intervals for each predicted mean. 195 participants (4870 observations) were included in this analysis.

Figure 3. A summary of the linear mixed models' results comparing the prevalence of each thought pattern between (i) Activities and (ii) Age-groups in the lockdown sample. On the lefthand side, there are the word clouds representing each thought pattern. Each word represents an experience-sampling item (see Table S1). Font size represents the magnitude of the loading, and the color describes the direction. Warm colors reflect positive loadings, while cool colors reflect negative loadings. The $y$-axis of each graph shows the predicted means for each thought pattern. The x-axis shows the five activity categories: (i) Working, (ii) Leisure activities, (iii) Social interactions, (iv) Media consumption and (v) Essential tasks (see Supplementary Information for details). White bars represent young participants, and grey bars represent older participants. Error bars represent the 95\% Confidence intervals for each predicted mean. 81 participants (1777 observations) were included in this analysis. 
A
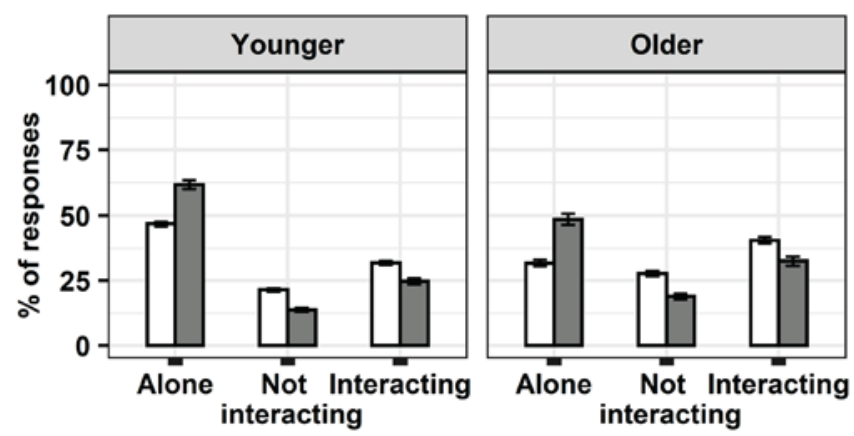

B

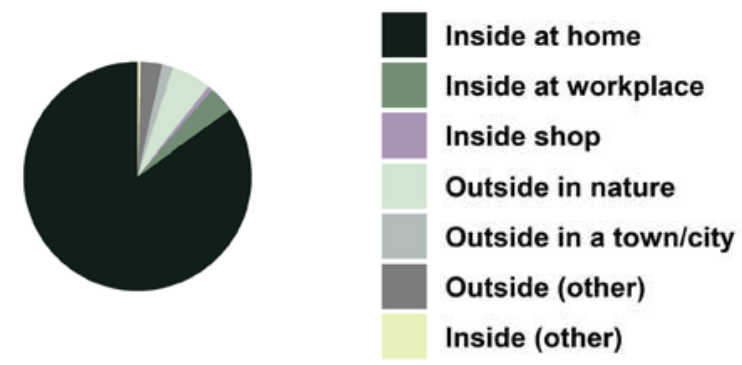
Inside at workplace

side shop

tside in nature Outside in a town/city Inside (other)

C

\begin{tabular}{|c|c|c|c|c|}
\hline \begin{tabular}{|c|} 
Rehearsing-Future \\
Controlled \\
Future-goals \\
Problem-Solving \\
Evolving \\
Future \\
Deliberate \\
Current-goals \\
Detailed \\
Important
\end{tabular} & $\begin{array}{c}\text { Current-goals } \\
\text { Conflicting } \\
\text { Positive } \\
\text { Wanted } \\
\text { Task } \\
\text { Past } \\
\text { Deliberate } \\
\text { Futaled } \\
\text { Future-goals } \\
\text { Distant-others }\end{array}$ & $\begin{array}{c}\text { Future-goals } \\
\text { Rehearsing-Future } \\
\text { Important } \\
\text { Close-others } \\
\text { Self } \\
\text { Future } \\
\text { Normal } \\
\text { Distantithers } \\
\text { vivid }\end{array}$ & $\begin{array}{c}\text { Distant-others } \\
\text { Detailed } \\
\text { Vivid } \\
\text { Images } \\
\text { Past } \\
\text { Evolving } \\
\text { Wanted } \\
\text { Task }\end{array}$ & $\begin{array}{c}\text { Rehearsing-Future } \\
\text { Current-goals } \\
\text { Task } \\
\text { Words } \\
\text { Imaines } \\
\text { Detailed } \\
\text { Normal } \\
\text { Evirniled }\end{array}$ \\
\hline $\begin{array}{l}\text { uture-alrectea } \\
\text { oblem-solving }\end{array}$ & & Epi & ery & Deta \\
\hline
\end{tabular}


Future-directed problem-solving

Current-goals

Evolving

Problem-Solving

Future-goals

Rehearsing-Future

Deliberate

Detailed
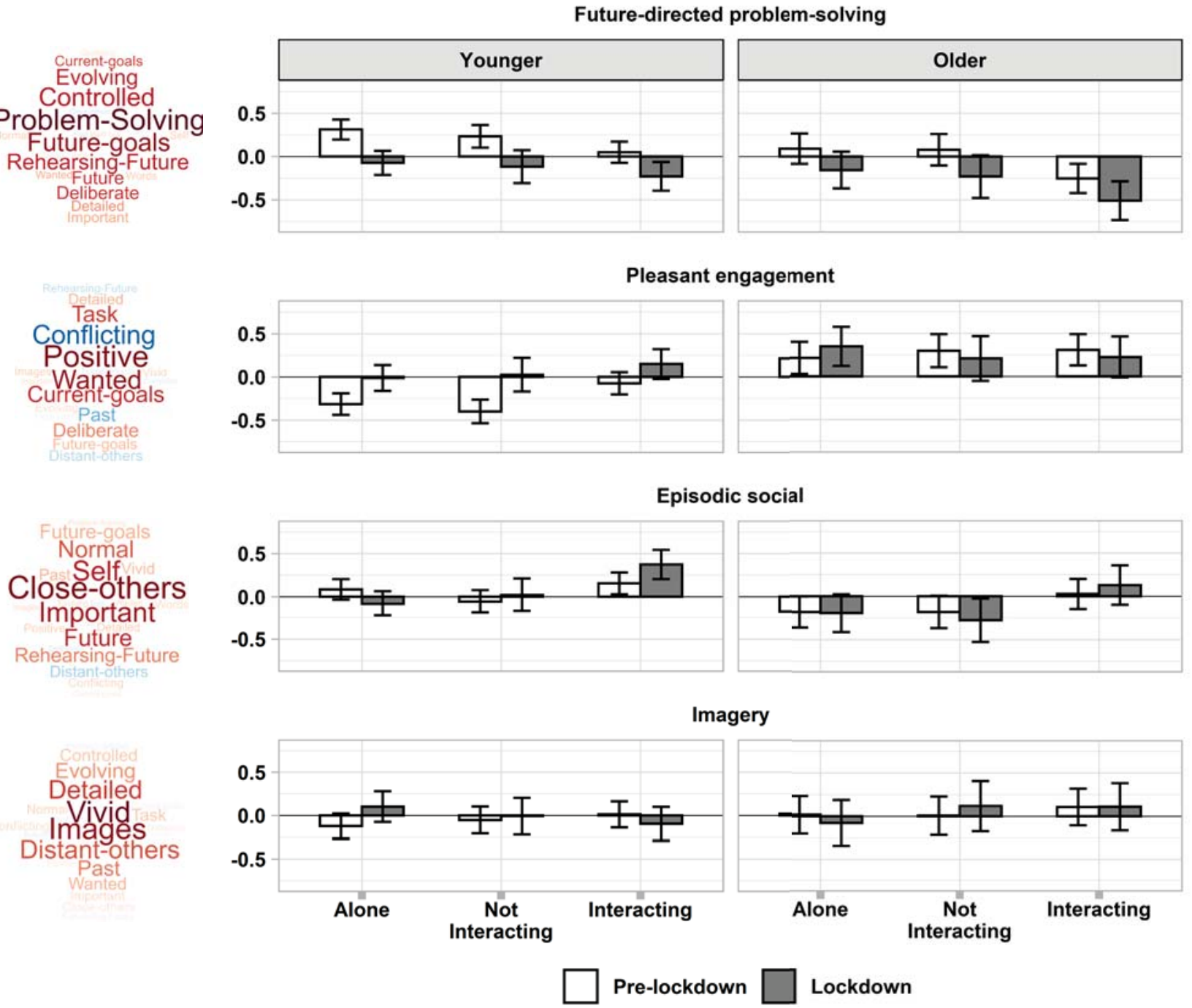

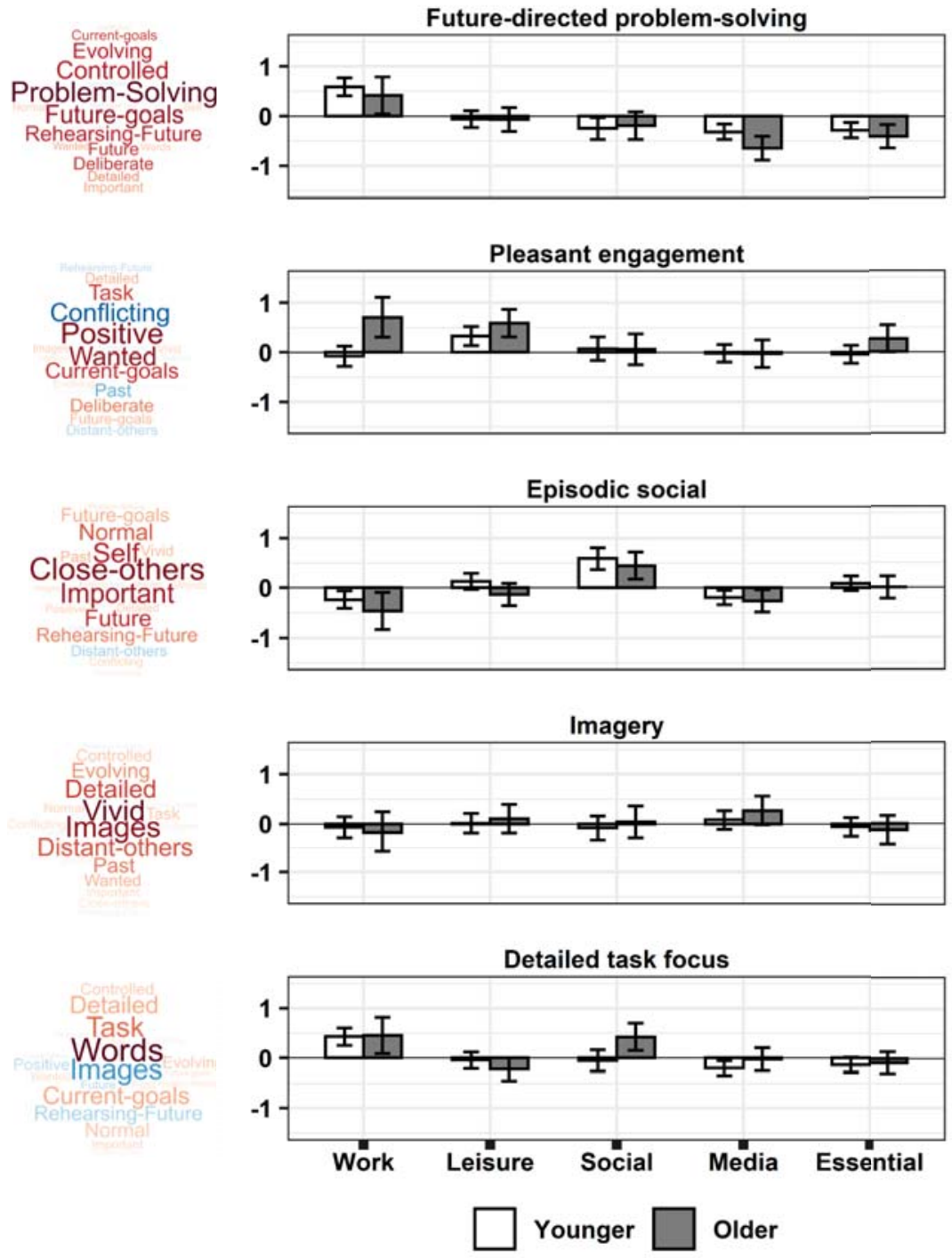